\title{
6. Layout of a COBOL Program
}

A COBOL program would often be written down on a coding sheet (see attached example); it is necessary when doing this - and later when keying the program in to the machine - to follow certain rules:

1. Most of the program should be written between columns 12 and 72 inclusive (known as Area B).

2. All Division names and Paragraph names and certain other headings should start between columns 8 and 11 inclusive (known as Area A).

3. Column 7 will usually be blank but if you want the line to be treated as a remark - an asterisk $\left(^{*}\right)$ should be placed in this column.

4. Columns $1-6$ and $73-80$ can generally be left blank.

COBOL Coding Form

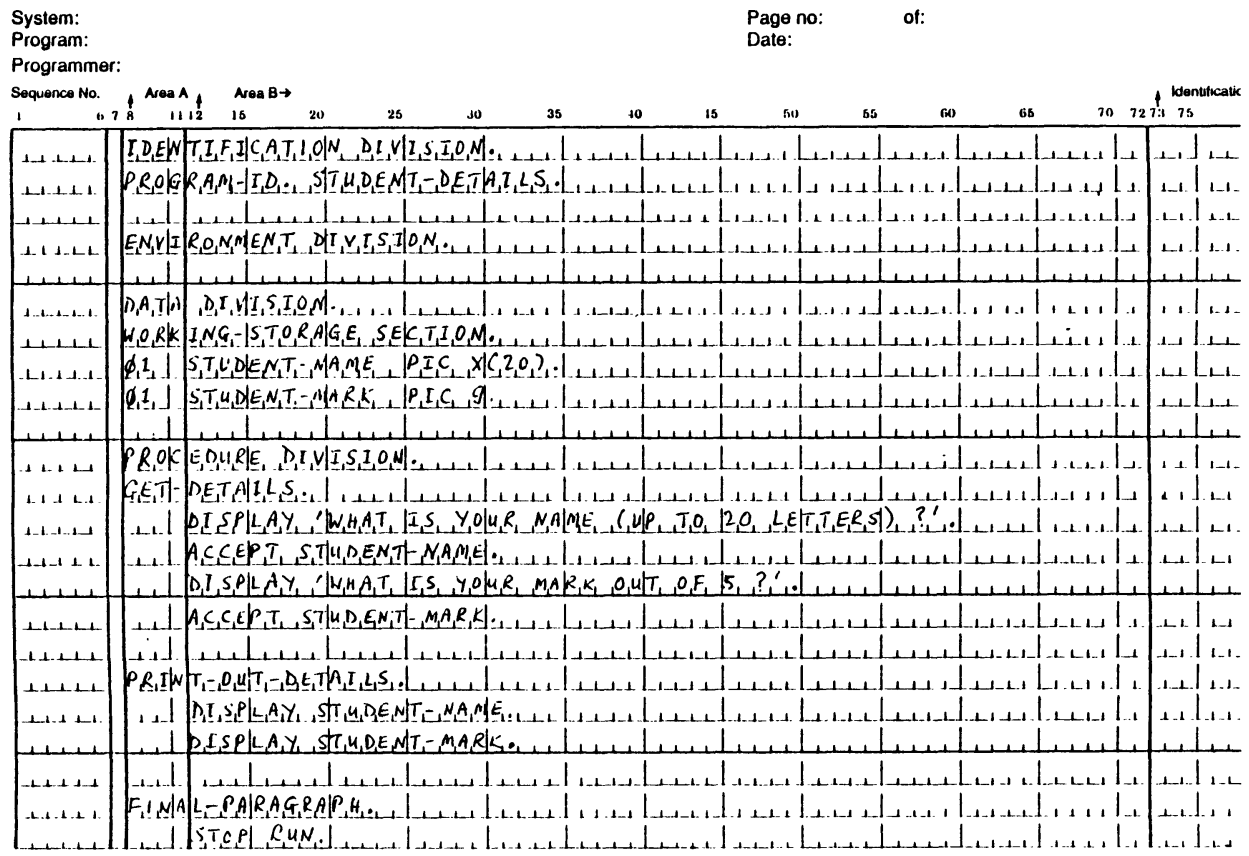

\section{Exercise}

1. Which items on the example program start in Area A ?

2. Write your answers to the exercises from lesson 5 correctly onto a

COBOL coding sheet. 